\title{
Conhecimento dos profissionais de saúde sobre eventos adversos em unidade de terapia intensiva
}

\author{
Health professionals' knowledge regarding adverse events in the intensive care unit \\ Conocimiento de los profesionales de salud sobre eventos adversos en unidad de cuidados intensivos
}

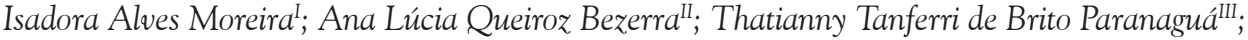 \\ Ana Elisa Bauer de Camargo Silva ${ }^{I V}$; Francino Machado de Azevedo Filho ${ }^{V}$
}

\begin{abstract}
RESUMO: O objetivo deste estudo foi analisar o conhecimento dos profissionais de saúde sobre a ocorrência de eventos adversos em unidade de terapia intensiva. Trata-se de estudo descritivo, realizado com 37 profissionais de saúde de duas unidades de terapia intensiva de um hospital de ensino da cidade de Goiânia, Goiás. A coleta de dados foi de janeiro a março de 2011, com instrumento estruturado e validado, sendo realizada análise estatística descritiva. Dos 37 profissionais, $45,9 \%$ desconheciam o significado de evento adverso, mas o reconheciam como parte do processo de trabalho. Relataram a ocorrência de 152 eventos durante o tempo de atuação na unidade. Para sua prevenção, foram sugeridas ações de educação continuada e organização do serviço. Os profissionais de saúde devem ser estimulados a notificá-los e as ações educativas devem ser simuladas a partir do contexto da prática em saúde, como um caminho para a assistência segura e de qualidade. Palavras-Chave: Enfermagem; unidades de terapia intensiva; segurança do paciente; iatrogenia.
\end{abstract}

ABSTRACT: This descriptive study to examine health professionals' knowledge of adverse events occurring in intensive care units was conducted with 37 health professionals from two intensive care units at a teaching hospital in Goiania, Goias State. Data were collected from January to March 2011 using a validated, structured instrument, and were treated with descriptive statistical analysis. Of the 37 health professionals, $45.9 \%$ were unaware of the significance of adverse events, but recognized them as part of the work process, and reported the occurrence of 152 events during their time working in the unit. Continuing professional development activities and service organization measures were suggested with a view to preventing adverse events. Health professionals should be encouraged to report adverse events, and educational actions should use simulation based on the practical healthcare context as a pathway to safe, quality care.

Keywords: Nursing; intensive care units; patient safety; iatrogenic disease.

RESUMEN: El estudio tuvo como objetivo analizar el conocimiento de los profesionales de salud sobre la ocurrencia de eventos adversos en unidad de cuidados intensivos. Se trata de un estudio descriptivo, realizado junto a 37 profesionales de la salud de dos unidades de cuidados intensivos de un hospital universitario de Goiânia, Goiás. La recolección de datos tuvo lugar de enero a marzo de 2011, con instrumento estructurado y validado. Ha sido realizado el análisis estadístico descriptivo. De los 37 profesionales, un 45,9\% desconocía el significado de evento adverso, sin embargo lo reconocía como parte del proceso de trabajo y relató la ocurrencia de 152 eventos durante el tiempo de actuación en la unidad. Para la prevención de eventos adversos, se han sugerido actividades de formación permanente y organización del servicio. Se debe alentar a los profesionales de salud a que informen eventos adversos y se deben simular acciones educativas dentro del contexto de la práctica en salud, como un camino hacia una atención segura y de calidad.

Palabras Clave: Enfermería; unidades de cuidados intensivos; seguridad del paciente; enfermedad iatrogénica.

\section{INTRODUÇÃO}

Nas instituições de saúde, os procedimentos realizados envolvem determinados riscos que são agravados na presença de falhas na estrutura e processos da assistência, tornando o cuidado inseguro e, como resultado, evidencia-se baixa qualidade no atendimento ao paciente e a ocorrência de incidentes.

Dentre os tipos de incidentes, os eventos adversos (EA) destacam-se por, obrigatoriamente, acarretar dano ao paciente, resultando em impacto significativo no sistema de saúde, incluindo pacientes, profissionais e instituições ${ }^{1}$.

Embora a busca pela segurança nas ações de saúde seja um desejo, a ocorrência de EA é uma realidade, inclusive em setores que prestam atendimento a pacientes críticos e que exigem alto padrão de qualidade - como nas unidades de terapia intensiva (UTI) ${ }^{2}$.

'Enfermeira do Hospital Universitário de Brasília, Brasil. E-mail: isadora.a.moreira@gmail.com.

IIProfessor Associado da Faculdade de Enfermagem da Universidade Federal de Goiás. Brasil. E-mail: analuciaqueiroz@uol.com.br.

IIIProfessor Adjunto da Faculdade de Ciências da Saúde da Universidade de Brasília. Brasil. E-mail: ttb.paranagua@gmail.com.

IVProfessor Adjunto da Faculdade de Enfermagem da Universidade Federal de Goiás. Brasil. E-mail: anaelisa@terra.com.br.

vDoutorando do Programa de Pós-Graduação em Enfermagem da Universidade de Brasília. Brasil. E-mail: francino21@gmail.com. 
Mundialmente, estima-se que os EA acometem 10\% dos pacientes admitidos em hospitais, podendo levar a uma debilidade temporária e até à morte ${ }^{3}$.

A publicação do relatório do Institute of Medicine, To error is human, de 1999, evidenciando que, anualmente, nos Estados Unidos, cerca de 98.000 pacientes morrem por eventos preveníveis ocorridos durante a hospitalização ${ }^{4}$, alavancou as pesquisas científicas quanto à segurança do paciente.

presente estudo objetivou analisar o conhecimento dos profissionais de saúde sobre a ocorrência de eventos adversos nas unidades de terapia intensiva de um hospital de ensino. Para tal, considerou-se que a redução dos EA implica a sua compreensão e, posteriormente, a elaboração de estratégias corretivas e preventivas, favorecendo uma assistência de qualidade e mais segura.

\section{REVISÃo DE LITERATURA}

Evento adverso é entendido como lesão ou dano não intencional, consequência do cuidado prestado, que resulta em incapacidade ou disfunção, temporária ou permanente, em prolongamento do tempo de permanência hospitalar ou morte. O que caracteriza o EA é o fato do dano ou lesão ter sido causado pelo cuidado prestado e não pela evolução da doença de base ${ }^{5}$.

Em UTI, a ocorrência de EA tem significado singular, por se tratar de um setor de alta complexidade. A prevalência de EA em UTI de hospitais universitários em Otawa é estimada em 20\% e evidencia-se que a cada cinco eventos, um é evitável ${ }^{6}$.

No Brasil, pesquisa aponta que, durante um período de quatro anos, 377 pacientes foram vítimas de EA, sendo que 22,3\% destes sofreram dois ou mais eventos, reforçando a necessidade de estabelecer protocolos de controle e prevenção?

Na região Centro-Oeste do Brasil, os primeiros estudos realizados no Estado de Goiás evidenciaram a ocorrência de EA vinculados, principalmente, ao processo de medicação ${ }^{8-10}$.

Outros EA frequentemente identificados em UTI estão relacionados às falhas na prevenção e diagnóstico de doenças, ao sistema de monitorização e interpretação dos monitores pelos profissionais, bem como às falhas durante a indicação, colocação, manutenção e retirada dos dispositivos tubulares ${ }^{11}$.

Em relação à assistência de enfermagem, destacam-se falhas na administração de medicamentos, no monitoramento de bombas de infusão, manuseio incorreto de materiais e artefatos terapêuticos e diagnósticos, além de falhas nas anotações de enfermagem ${ }^{12}$.

Considerando que a equipe de enfermagem perfaz maior contingente de pessoal nas instituições hospitalares, que a expectativa da assistência prestada numa UTI é garantir o melhor resultado dentro das condições clínicas e da gravidade dos pacientes com menores índices possíveis de complicações decorrentes dos procedimentos realizados e que o alcance da qualidade da assistência exige adequação e controle dos processos e estrutura do serviço, há a necessidade de investigar a percepção da equipe multiprofissional que presta atendimento ao paciente crítico ${ }^{13}$.

Nessa perspectiva, conhecer os EA possibilita a identificação de falhas latentes nas diversas etapas do processo do cuidar, o que contribui para a realização de estratégias que reduzam e interceptem essas falhas, minimizando seu impacto e conduzindo o profissional a uma prática segura ${ }^{14}$.

\section{Metodologia}

Estudo descritivo, desenvolvido nas UTI médica e cirúrgica de um hospital universitário da Região Centro-Oeste, Brasil. Atualmente, a UTI possui uma capacidade física para 14 leitos, sendo seis deles na UTI médica e oito, na cirúrgica.

Todos os profissionais foram convidados a participar do estudo, constituindo uma população de 104 indivíduos, incluindo médicos, enfermeiros e técnicos de enfermagem. Foram excluídos os profissionais que estavam em licença durante o período de coleta de dados e aqueles que não foram encontrados em até três visitas programadas na UTI.

A coleta de dados foi realizada com 37 profissionais, entre os meses de janeiro a março de 2011. Utilizou-se a técnica de entrevista norteada por instrumento estruturado, avaliado por experts e submetido ao teste-piloto.

$\mathrm{O}$ instrumento consta de três partes. A primeira investiga dados de caracterização dos participantes: idade, sexo, categoria profissional, tempo de formação e de atuação (na profissão, instituição e na unidade), carga horária e turno de trabalho na instituição, vínculos empregatícios e educação continuada. A segunda contém questões sobre o conhecimento dos profissionais acerca do EA, medidas preventivas e gestão de riscos na unidade. A terceira consta dos tipos de EA identificados pelos profissionais durante a atuação na UTI, as consequências para o paciente, condutas clínicas e administrativas adotadas e conhecimento do paciente/família sobre o ocorrido.

Os relatos dos participantes foram transcritos em documento do Microsoft Word para formar o corpus de análise, sendo identificados pela letra E, seguido de um número cardinal, conforme a sequência das entrevistas. Os dados quantitativos foram inseridos em planilha eletrônica do programa Microsoft Excel, versão 2007. Em seguida, foi realizada análise estatística descritiva das variáveis categóricas e média para as variáveis contínuas. Os resultados foram apresentados em tabelas e discutidos com base na literatura. 
Os aspectos éticos seguem a Resolução CNS n ${ }^{\circ}$ 196/96, sendo o estudo vinculado ao projeto Análise de ocorrências de eventos adversos em um hospital da Rede Sentinela da Região Centro-Oeste, sob Protocolo $\mathrm{n}^{\mathrm{o}}$ 064/2008.

\section{Resultados e Discussão}

\section{Caracterização dos profissionais}

Participaram do estudo 37 profissionais, sendo 23 da UTI médica e 14 da UTI cirúrgica. Aqueles profissionais que não foram encontrados em até três tentativas foram excluídos da pesquisa. Os dados de caracterização dos participantes estão informados na Tabela 1.

Entre os profissionais, 20(54,1\%) eram técnicos de enfermagem, seguidos de enfermeiros, com 13(35,1\%), e médicos, com 4(10,8\%). O sexo masculino foi predominante, com 21(62,2\%) e a idade variou entre 21 e 59 anos, predominando a faixa etária de 31 a 40 anos, em $16(43,2 \%)$ profissionais.

TABELA 1: Caracterização dos profissionais das UTI médica e cirúrgica de um hospital de ensino. Goiânia, 2011. ( $N=37)$

\begin{tabular}{|c|c|c|}
\hline Variáveis & $f$ & $\%$ \\
\hline \multicolumn{3}{|l|}{ Categoria profissional } \\
\hline Técnico de enfermagem & 20 & 54,1 \\
\hline Enfermeiro & 13 & 35,1 \\
\hline Médico & 4 & 10,8 \\
\hline \multicolumn{3}{|l|}{ Sexo } \\
\hline Feminino & 16 & 43,2 \\
\hline Masculino & 21 & 56,8 \\
\hline \multicolumn{3}{|l|}{ Idade (Média = 38 anos) } \\
\hline $21-30$ & 7 & 18,9 \\
\hline $31-40$ & 16 & 43,2 \\
\hline $41-50$ & 11 & 29,7 \\
\hline $51-59$ & 3 & 8,1 \\
\hline \multicolumn{3}{|l|}{ Tempo de formado } \\
\hline Menos de 1 ano & 3 & 8,1 \\
\hline De 1 a 10 anos & 14 & 37.8 \\
\hline De 11 a 20 anos & 18 & 48,7 \\
\hline Acima de 20 anos & 2 & 5,4 \\
\hline \multicolumn{3}{|c|}{ Tempo de atuação na unidade } \\
\hline Menos de 1 ano & 3 & 8,1 \\
\hline De 1 a 10 anos & 11 & 29,7 \\
\hline De 11 a 20 anos & 18 & 48,6 \\
\hline Mais de 20 anos & 3 & 8,1 \\
\hline Não responderam & 2 & 5,4 \\
\hline \multicolumn{3}{|l|}{ Carga horária na unidade } \\
\hline 20 horas/semana & 2 & 5,4 \\
\hline 30 horas/semana & 31 & 83,7 \\
\hline 40 horas/semana & 1 & 2,7 \\
\hline 60 horas/semana & 3 & 8,1 \\
\hline \multicolumn{3}{|l|}{ Vínculos Empregatícios } \\
\hline Só nessa unidade & 17 & 45,9 \\
\hline Mais de um vínculo & 20 & 54,1 \\
\hline
\end{tabular}

O tempo de formado foi de 11 a 20 anos para $18(48,6 \%)$ profissionais e o tempo de atuação na unidade superior a 11 anos também para $18(48,6 \%)$ deles. A carga horária semanal de trabalho na unidade, de parte dos profissionais, ficou entre 20 a 60 horas e 20(54\%) relataram possuir mais de um vínculo empregatício.

\section{Conhecimento dos profissionais sobre even-} tos adversos

Os profissionais demonstraram conhecimento sobre o significado de EA, porém, com superficialidade, segundo $17(45,94 \%)$ relatos, como, por exemplo:

Algo que acontece fora do normal com o paciente. (E1)

Algo ocorrido que não é previsto para o paciente. (E16)

O desconhecimento - ou, ainda, o conhecimento errôneo - foi constatado em 20(54,05\%) dos relatos, como, por exemplo:

Qualquer coisa que ocorra dentro do ambiente de trabalho. (E11)

Não sei. (E09)

O conhecimento limitado dos profissionais sobre os EA evidencia a necessidade de políticas gerenciais e desenvolvimento de ações estratégicas voltadas para a educação continuada da equipe multiprofissional, no sentido de promover melhorias na qualidade e segurança no processo de cuidar. A educação em serviço permite uma compreensão dos EA, facilitando, assim, a investigação da qualidade da assistência e, também, uma melhora no serviço oferecido ao paciente $e^{12,15}$.

O investimento na formação continuada mantém relação direta com a melhoria da capacidade de rendimento para o trabalho e pode auxiliar na prevenção dos $\mathrm{EA}^{16}$. Salienta-se, ainda, a importância de levar ao conhecimento dos profissionais de saúde os resultados encontrados nos estudos sobre EA, pois o entendimento sobre a sua etiologia e contribuição do sistema hospitalar apresenta resultados significativos ${ }^{17}$.

\section{Comunicação de eventos adversos}

Em relação à comunicação da ocorrência de EA, apenas $14(37,83 \%)$ profissionais afirmaram relatar verbalmente para o enfermeiro ou médico supervisor da unidade. No entanto, nenhum dos profissionais notifica ao Serviço de Gerenciamento de Riscos tais eventos nem possui orientação sistematizada informá-los. Paradoxalmente, os mesmos profissionais que comunicam verbalmente o EA ao enfermeiro ou ao médico informaram que registram o evento no prontuário do paciente.

O registro é considerado ação fundamental na obtenção de informações de falhas e possibilita a implantação de medidas voltadas para a redução do número dessas ocorrências ${ }^{18}$.

As anotações em prontuários ou registro de informações nos serviços de saúde têm valor significativo, são fontes de investigação, educação e documento legal. São 
consideradas objeto de avaliação da assistência prestada ao paciente e da qualidade das anotações elaboradas pela equipe multiprofissional. Porém é importante que se estimule a notificação sistemática por meio de um instrumento padronizado e que, preferencialmente, seja eletrônico, a fim de facilitar e agilizar o processo de comunicação e análise dos eventos ocorridos ${ }^{19,20}$.

\section{Tipo de eventos adversos}

Foram relatados $152 \mathrm{EA}$ vivenciados pelos profissionais de saúde durante o período de atuação na UTI. Os tipos de EA estão descritos na Tabela 2.

TABELA 2: Eventos adversos ocorridos nas UTI médica e cirúrgica de um hospital de ensino. Goiânia, 2011.

\begin{tabular}{lcc}
\hline Eventos adversos & $\mathrm{f}$ & $\%$ \\
\hline Cateter, sondas e drenos & 35 & 23,0 \\
Infecção hospitalar & 24 & 15,8 \\
Úlcera por pressão & 20 & 13,1 \\
Processos alérgicos & 17 & 11,2 \\
Complicações cirúrgicas & 17 & 11,2 \\
Erro de medicação & 14 & 9,2 \\
Queda & 9 & 5,9 \\
Incompatibilidade de hemoderivados & 5 & 3,2 \\
Queimadura & 4 & 2,6 \\
Hemorragias & 3 & 2,0 \\
Falha durante procedimentos técnicos & 1 & 0,7 \\
Sepse & 1 & 0,7 \\
Omissão de cuidado & 1 & 0,7 \\
Tentativa de autoextermínio & 1 & 0,7 \\
Total & 152 & $\mathbf{1 0 0 , 0}$ \\
\hline
\end{tabular}

Do total de EA vivenciados, 35(23\%) foram relacionados aos dispositivos tubulares, sendo um evento frequente na literatura ${ }^{10}$. Considerando que a terapia intravenosa é um dos procedimentos mais realizados, a inserção, manutenção e retirada indevida desses dispositivos predispõe a ocorrência de EA, sendo mais frequente a infecção e o extravasamento sanguíneo $^{11,21}$. As causas dos EA foram 17 obstruções, 13 retiradas não programadas dos dispositivos e cinco fixações inadequadas.

Dentre as consequências, 14 exigiram monitoramento; 16 contribuíram ou resultaram em dano temporário e prolongaram a internação e quatro contribuíram ou resultaram em dano temporário, requerendo intervenção.

Apesar de parecer simples, o procedimento de sondagem está sujeito a graves complicações, interferindo na continuidade da assistência ${ }^{22}$, da mesma forma que a retirada indevida de drenos pode trazer sérios riscos ao paciente, devendo ter avaliação criteriosa por profissional habilitado ${ }^{23}$.
A redução de EA relacionados aos dispositivos tubulares está associada às orientações à equipe de saúde quanto à técnica de inserção e manutenção, cuidados durante transferências do paciente, além de orientar pacientes e familiares sobre a importância da manutenção do dispositivo ${ }^{10}$.

Referente à infecção hospitalar, foram relatados $24(15,8 \%)$ EA, 11 relacionados à infecção do sítio cirúrgico, quatro no trato urinário pós-sondagem vesical, quatro infecções sanguíneas por acesso vascular, duas no local de inserção de dispositivos tubulares, duas pneumonias e uma infecção de ferida não operatória.

Quanto às consequências para o paciente, um requereu monitoramento; 15 contribuíram ou resultaram em dano temporário e demandaram intervenção, dois resultaram em dano permanente e prolongaram a internação, uma causou dano e necessitou de intervenção para manutenção da vida e cinco resultaram em óbito.

A infecção hospitalar, além de prolongar o tempo de internação, traz graves consequências ao paciente e alto custo ao sistema de saúde. As instituições de saúde devem elaborar protocolos de assistência ao paciente, prover recursos materiais e capacitar toda a equipe no sentido de reduzir a ocorrência da infecção hospitalar e obter melhores indicadores de saúde 24 .

Foram registrados $20(13,1 \%)$ EA relacionados à úlcera por pressão, que resultaram em dano, prolongaram a internação e requereram intervenção adicional ao tratamento inicial.

As úlceras por pressão destacam-se por serem eventos preveníveis pela adoção de medidas preventivas como monitoramento, inspeção e proteção da pele, além de mudança de decúbito do paciente. $\mathrm{O}$ profissional de saúde precisa estar atento aos problemas que as úlceras por pressão podem ocasionar ao paciente, à família e à instituição hospitalar. Estas ocorrências prolongam a hospitalização, dificultam a recuperação do paciente, aumentam o risco para o desenvolvimento de complicações e representam um acréscimo no sofrimento físico e emocional dos clientes ao reduzir a sua independência na realização das atividades, além de aumentar os custos ${ }^{25}$.

Foram relatados $17(11,2 \%)$ processos alérgicos, dos quais 12 estavam relacionados a medicamentos e cinco ao uso de esparadrapo. Dipirona foi o medicamento com mais relato de processo alérgico - seis casos -, seguido da Vancomicina, com quatro. Como consequências dos processos alérgicos, seis requereram monitoramento e 11 resultaram em dano temporário com intervenção.

É de grande relevância a notificação de reações alérgicas a produtos médico-hospitalares para o serviço de gerenciamento de risco, uma vez que é possível gerenciar os produtos por meio das ações de farmacovigilância ${ }^{26,27}$. Essas notificações resultaram no monitoramento e avaliação desses produtos, o que contribui para a qualidade da assistência. 
Nas complicações cirúrgicas, foram relatados $17(11,2 \%)$ casos, sendo 11 de pneumotórax, quatro de embolia/tromboembolia e uma parada cardiorrespiratória.

Quanto às consequências, 13 requereram monitoramento, três resultaram em dano permanente e prolongaram o tempo de internação e uma resultou na morte do paciente. Para evitar as complicações decorrentes da estimativa de 234 milhões de cirurgias realizadas anualmente, a Aliança Mundial para Segurança do Paciente propôs o desafio Cirurgias seguras salvam vidas, o qual recomenda o uso de protocolos no perioperatório para reduzir a morbimortalidade relacionada a esses procedimentos ${ }^{3}$.

Foram apontados 14(9,2\%) EA relacionados à medicação, conforme Tabela 3.

TABELA 3: Tipos de erros de medicação ocorridos nas UTI médica e cirúrgica de um hospital de ensino. Goiânia, 2011.

\begin{tabular}{lcc}
\hline Erros de medicação & f & $\%$ \\
\hline Dose & 5 & 35,7 \\
Troca de paciente & 2 & 14,3 \\
Suspensão do medicamento por falta & 2 & 14,3 \\
Horário & 1 & 7,1 \\
Omissão & 1 & 7,1 \\
Técnica & 1 & 7,1 \\
Medicamento errado & 1 & 7,1 \\
Checagem inadequada & 1 & 7,1 \\
Total & 14 & 100,0 \\
\hline
\end{tabular}

Entre os eventos citados, $9(64,2 \%)$ estavam relacionados aos cinco certos na administração de medicamentos (paciente, medicamento, dose, horário e registros certos), o que evidencia que esse simples processo de verificação não está sendo seguido. Quanto às consequências, nove requereram monitoramento $\mathrm{e}$ cinco resultaram em dano temporário com intervenção.

Estima-se que pelo menos 1,5 milhão de pessoas, nos Estados Unidos, sejam atingidas por erros de medicação, anualmente, gerando custos superiores a 3,5 bilhões de dólares ${ }^{3}$.

As instituições de saúde devem elaborar um sistema seguro de medicação que auxilie os profissionais na prevenção dos erros, por meio de medidas facilitadoras para o processo de medicação e que dificulte ocorrências de erros ${ }^{28}$.

Os profissionais relataram 9(5,9\%) quedas do leito, entre as quais sete requereram monitoramento e duas resultaram em dano temporário com intervenção. Ver Tabela 2.

A queda do paciente é um EA frequente decorrente de vários fatores como a falta de vigilância necessária e a capacidade ineficiente do paciente em deambular sem auxílio. Sua ocorrência pode ocasionar lesões e prejudicar a integridade física e emocional dos mesmos além do aumento de custos hospitalares e até a morte ${ }^{29}$.

Várias medidas podem ser adotadas para evitar que quedas ocorram, como elevação de grades nas camas e a aplicação de técnicas de restrição de movimentos. A contenção no leito é uma medida adequada para os pacientes agitados desde que realizada corretamente e não acarrete prejuízos, devendo ser avaliado e monitorado com frequência pela equipe para determinar a continuidade ou não do procedimento ${ }^{29}$.

Foram 4(2,6\%) casos de queimadura, sendo dois relacionados ao uso do desfibrilador, um ao uso de compressa quente e um ao bisturi elétrico, conforme Tabela 2. As consequências desses eventos contribuíram para ou resultaram em dano temporário com intervenção.

A queimadura deixa sequelas na área lesada e, especialmente com compressas quentes, ocorrem por desatenção profissional à temperatura da água e ao tempo de exposição na pele do paciente. Casos de queimaduras relacionadas a equipamentos também são citados por diversos autores, devendo a causa ser investigada ${ }^{11,12}$ para subsidiar a elaboração de bundles direcionados a procedimentos que podem expor o paciente a esse risco. Os bundles têm sido apontados como uma medida de impacto que direciona a adoção de práticas seguras e seu uso deve ser estimulado em ambientes que primam pela excelência do cuidado ${ }^{30}$.

Foram relatadas $5(3,2 \%)$ infusões de hemoderivado com incompatibilidade sanguínea que requereram monitoramento. Para a prática transfusional, é necessário que os profissionais estejam qualificados e conheçam todos os incidentes relacionados ao procedimento, como incompatibilidade sanguínea, um dos EA mais graves. Além disso, a documentação das informações e organização de dados clínicos do paciente devem estar organizados adequadamente, a fim de otimizar o serviço, prever as possibilidades de incidentes e, ainda, introduzir medidas de correção e prevenção de erros, o que contribui para melhor segurança na prática de transfusão de hemoderivados ${ }^{31}$.

Outros eventos foram relatados, como 3(42,9\%) hemorragias, $1(14,3 \%)$ falha técnica durante procedimento, 1(14,3\%) sepse, 1(14,28\%) omissão de cuidado e $1(14,3 \%)$ tentativa de autoextermínio. Quanto às consequências desses EA, três ocasionaram dano temporário com intervenção; um requereu monitoramento, um resultou em dano temporário com monitoramento e dois converteram-se em óbito.

Constata-se que os profissionais de saúde convivem com a ocorrência dos EA nas UTI; contudo, o conhecimento sobre a temática é limitado, ao passo que o relato de presenciar o incidente é expressivo. 


\section{Condutas frente ao evento adverso}

Em relação às condutas frente à ocorrência dos EA, $130(85,5 \%)$ resultaram em medidas simples como observação e intervenções com antibioticoterapia, curativos, monitoramento, drenagem, suspensão do medicamento e uso de anti-histamínico. Não foram relatadas condutas para 22(14,5\%) EA. A comunicação do EA ao paciente ocorreu em $67(44,1 \%)$ casos. $\mathrm{O}$ empoderamento do paciente para garantir sua própria segurança tem sido estimulado e uma das ações inclui a comunicação ao paciente para que ele auxilie na tomada de decisão, de modo que as consequências sejam minimizadas da melhor forma possível ${ }^{32}$.

Entre as condutas administrativas em relação ao profissional envolvido no EA, investigadas no presente estudo, 21(13,8\%) ocorrências resultaram em advertência verbal, esclarecimento e orientação acerca dos motivos do erro, comunicação ao supervisor e anotação na evolução do paciente.

Como condutas gerenciais e educativas na prevenção de 42(27,6\%) EA, foram citadas educação continuada, fiscalização e orientação do profissional.

Quanto à existência de condutas preventivas de EA adotadas na instituição e nas UTI, 16(43,2\%) profissionais referiram a realização de cursos e treinamentos com a Comissão de Controle de Infecção Hospitalar. Referiram medidas administrativas, como protocolos de procedimentos e rotinas, orientação sobre fluxo, preparo e administração de medicamentos entre farmácia e unidades clínicas e a sistematização da assistência de enfermagem $6(27,3 \%)$ profissionais.

Como sugestões para evitar o EA, 23(62,1\%) profissionais citaram as atividades educativas, sendo $15(40,5 \%)$ relacionadas ao treinamento, orientação e educação continuada e $8(21,6 \%)$ à segurança e atenção do profissional. Quanto à organização do serviço, 4(10,8\%) profissionais sugeriram a melhoria na estrutura física e no sistema de informação/comunicação interna da unidade e instituição.

A ocorrência de EA interfere na segurança do paciente e pode ser influenciada pela gestão do serviço devido às falhas nas etapas do planejamento, controle e supervisão do trabalho quanto às demandas na previsão, provisão e manutenção dos equipamentos e recursos materiais ${ }^{33,34}$.

Ter conhecimento desses aspectos é relevante como medida preventiva, permitindo a cada profissional a identificação e avaliação dos riscos, além de promover a conscientização coletiva para o cuidado seguro ${ }^{20}$.

Desse modo, autores enfatizam que a prática de ações educativas junto aos profissionais envolvidos diretamente na assistência poderá favorecer a melhoria da qualidade do cuidado prestado ao cliente e prevenir a repetição do erro, desenvolvendo, assim, uma cultura de segurança ${ }^{18,35}$.

O apoio da gestão hospitalar para a segurança do paciente é uma dimensão necessária para a transformação da prática assistencial, o que implica a necessidade de uma atitude mais proativa por parte dos gestores e da comunicação entre os diferentes subsistemas que compõem os serviços de saúde ${ }^{36}$. Entretanto, ressalta-se que a segurança do paciente deve ser responsabilidade de todos os profissionais envolvidos no processo do cuidar, subsidiados por programas de desenvolvimento e qualificação profissional, tendo a educação como uma prática essencial para a segurança do paciente $^{37}$.

\section{Conclusão}

Constatou-se que os profissionais possuem conhecimento superficial sobre o conceito dos EA, mas identificam e reconhecem o evento como parte da assistência à saúde quando esta não é realizada com qualidade.

Evidenciou-se a subnotificação de eventos no contexto da prática e a existência de lacunas nos processos educativos institucionais, demonstrando fragilidades na segurança do paciente.

Sugerem-se melhorias na gestão dos processos de trabalho e do sistema organizacional, além do fomento das bases para o desenvolvimento de evidências clínicas visando à construção de conhecimentos e atitudes para a prevenção de EA e promoção da cultura de segurança. Por ser um ambiente onde as atividades são complexas, os gestores das UTI devem adotar políticas direcionadas à abordagem sistemática e aprendizagem a partir dos erros no sentido de capacitar os profissionais para serem proativos frente às ocorrências de eventos, a fim de reduzir as consequências ao paciente.

O estudo apresenta limitações quanto ao número de profissionais, especialmente da equipe médica, visto que poucos aceitaram participar do estudo. Acredita-se que a utilização de um método de coleta de informações on line possa expandir a população elegível de estudos futuros, além de subsidiar a elaboração de um sistema de informação local que facilite a compilação dos dados, estimule os registros dos EA pelos profissionais e auxilie a tomada de decisão dos gestores.

\section{REFERÊNCIAS}

1.Oliveira RB. Eventos adversos com medicamentos favorecidos pelo sistema de medicação de um hospital público no município do Rio de Janeiro [dissertação de mestrado]. Rio de Janeiro: Universidade Federal do Estado do Rio de Janeiro; 2010.

2.Bohomol E, Ramos LH, D'Innocenzo M. Medication errors in an intensive care unit. J Adv Nurs. 2009; 65:1259-67.

3.World Health Organization (WHO). World Alliance for Patient Safety. Global Patient Safety Challenge: 2006-2007. 2006 [cited in 2014 Apr 25] Available from: http://www.who.int/patientsafety/information_centre/ WHO_EIP_HDS_PSP_2006.1.pdf.

4.Kohn LT, Corrigan J, Donaldson MS. To err is human: building a safer health system: National Academy Press; 2000. 
5.World Health Organization (WHO). Conceptual framework for the International Classification for Patient Safety. Final Technical Report. Geneva, 2009 [cited 2015 Mar 08]. Available from: http://www.who.int/patientsafety/taxonomy/icps_full_report.pdf.

6.Forster AJ, Kyeremanteng K, Hooper J, Shojania KG, van Walraven $\mathrm{C}$. The impact of adverse events in the intensive care unit on hospital mortality and length of stay. BMC Health Serv Res. 2008; 8:259.

7.Toffoletto MC. Fatores associados aos eventos adversos em unidade de terapia intensiva [tese de doutorado] São Paulo: Universidade de São Paulo; 2008.

8.Silva AEBC, Reis AMM, Miasso AI, Santos JO, Cassiani SHDB. Adverse drug events in a sentinel hospital in the State of Goiás, Brazil. Rev Latino-Am Enfermagem. 2011; 19:378-86.

9.Bezerra ALQ, Silva AEBC, Branquinho NCSS, Paranaguá TTB. Análise de queixas técnicas e eventos adversos notificados em um hospital sentinela. Rev enferm UERJ. 2009; 17:467-72.

10.Carneiro FS, Bezerra ALQ, Silva AEBC, Souza LP, Paranaguá TTB, Branquinho NCSS. Eventos adversos na clínica cirúrgica de um hospital universitário: instrumento de avaliação da qualidade. Rev enferm UERJ. 2011; 19:204-11.

11.Canineu R, Guimarães HP, Lopes RD, Vendrame LS, Fonseca Júnior MA, Lopes AC. Iatrogenia em medicina intensiva. Rev bras ter intensiva. 2006; 18:95-8.

12.Beccaria LM, Pereira RAM, Contrin LM, Lobo SMA, Trajano DHL. Eventos adversos na assistência de enfermagem em uma unidade de terapia intensiva. Rev bras ter intensiva. 2009; 21:276-82.

13.Belela ASC, Peterlini MAS, Pedreira MLG. Revelação da ocorrência de erro de medicação em unidade de cuidados intensivos pediátricos. Rev bras ter intensiva. 2010; 22:257-63.

14.Chaboyer W, Thalib L, Foster M, Ball C, Richards B. Predictors of adverse events in patients after discharge from the intensive care unit. Am J Crit Care. 2008; 17:255-63. 15.Vituri DW, Cacciari P, Gvozd R, Kuwabara CCT, Cardoso MGP. Indicadores de qualidade como estratégia para melhoria da qualidade do cuidado em um hospital universitário. Cien Cuid e Saúde. 2010; 9(4):782-90. 16.Azevedo Filho FM, Martins IMS, Soares CSRS, Fazendeiro PG, Paranaguá TTB, Bezerra ALQ. Administración de medicamentos: conocimiento de los enfermeros del sector de urgencia y emergencia. Enferm glob. 2012; 11:54-69. 17.Szekendi MK, Sullivan C, Bobb A, Feinglass J, Rooney $\mathrm{D}$, Barnard C, et al. Active surveillance using electronic triggers to detect adverse events in hospitalized patients. Qua saf health care. 2006; 15(3):184-90.

18.Claro CM, Krocockz DVC, Toffolleto MC, Padilha KG. Eventos adversos em Unidade de Terapia Intensiva: percepção dos enfermeiros sobre a cultura não punitiva. Rev Esc Enferm USP. 2011; 45:167-72.

19.Paiva MCMS, Paiva SAR, Berti HW. Eventos adversos: análise de um instrumento de notificação utilizado no gerenciamento de enfermagem. Rev esc enferm USP. 2010; 44:287-94.

20.Setz VG, D’Innocenzo M. Avaliação da qualidade dos registros de enfermagem no prontuário por meio da auditoria. Acta Paul Enferm. 2009; 22:313-7.

21.Machado AF, Pedreira MLG, Chaud MN. Adverse events related to the use of peripheral intravenous catheters in children according to dressing regimens. Rev Latino-Am Enferm. 2008; 16:362-7.

22.Nascimento CCP, Toffoletto MC, Gonçalves LA, Freitas WG, Padilha KG. Indicators of healthcare results: analysis of adverse events during hospital stays. Rev Latino-Am Enferm. 2008; 16:746-51.

23.Parra AV, Amorim RC, Wigman SE, Baccaria LM. Retirada de dreno torácico em pós operatório de cirurgia cardíaca. Arq Ciênc Saúde. 2005; 12 (2):116-9.

24.Bezerra ALQ, Queiroz E, Weber J, Paranagua T. Reacciones adversas: indicadores de resultados según la percepción de las enfermeras de un hospital centinela. Enferm glob. 2012; 11:186-97.

25.Silva RCL, Figueiredo NMA, Meirelles IB, organizadores. Feridas: fundamentos e atualizacoes em enfermagem. $3^{a}$ ed. São Caetano do Sul: Yendis; 2011.

26.Romeu GA, Távora MRF, Costa AKM, Souza MOB, Gondim APS. Notificacao de reacoes adversas em um hospital sentinela de Fortaleza - Ceara. R Bras Farm Hosp Serv Saúde São Paulo. 2011; 2(1):5-9.

27. Organização Mundial da Saúde. Monitorização da segurança de medicamentos: diretrizes para criação e funcionamento de um Centro de Farmacovigilância. Brasília (DF): Organização Pan-Americana da Saúde, 2005. 28.Silva AEBC, Cassiani SHB. Erros de medicação em hospital universitário: tipo, causas, sugestões e providências. Rev bras enferm. 2004; 57:671-4.

29.Santos JC, Ceolim MF. Iatrogenias de enfermagem em pacientes idosos hospitalizados. Rev esc enferm USP. 2009; 43:810-7.

30.Shimabukuro PMS, Paulon P, Feldman LB. Implantação de bundles em unidade de terapia intensiva: um relato de experiência. Rev Enferm UFSM. 2014; 4(1):227-36. 31.Curcioli ACJV, Carvalho EC. Infusion of hematopoietic stem cells: types, characteristics, adverse and transfusion reactions and the implications for nursing. Rev Latino-Am Enferm. 2010; 18:716-24.

32.Lyons M. Should patients have a role in patient safety? A safety engineering view. Qua saf health care. 2007; 16(2):140-2.

33.Azzolin G, Peduzzi M. Processo de trabalho gerencial e processo de enfermagem na perspectiva de docentes de enfermagem. Rev Gaúcha Enferm. 2008; 28:549-55. 34.Garcia SD, Haddad MCL, Dellaroza MSG, Costa DB, Miranda JM. Gestão de material médico-hospitalar e o processo de trabalho em um hospital público. Rev Bras Enferm. 2012; 65:339-46.

35. Oliveira NC, Chaves LDP. Gerenciamento de recursos materiais: o papel da enfermeira de unidade de terapia intensiva. Rev Rene. 2009;10(4):19-27.

36. Mello JF, Barbosa SFF. Cultura de segurança do paciente em terapia intensiva: recomendações da enfermagem. Texto contexto-enferm. 2013; 22:1124-33 37.Lima FDM. Patient Safety and Interventions for quality in health care. Rev Espaço para a Saúde. 2014;15(3):22-9. 